\title{
Neural Stem Cells Improve Memory in an Inducible Mouse Model of Neuronal Loss
}

\author{
Tritia R. Yamasaki, Mathew Blurton-Jones, Debbi A. Morrissette, Masashi Kitazawa, Salvatore Oddo, and \\ Frank M. LaFerla \\ Department of Neurobiology and Behavior, University of California, Irvine, Irvine, California 92697
}

\begin{abstract}
Neuronal loss is a major pathological outcome of many common neurological disorders, including ischemia, traumatic brain injury, and Alzheimer disease. Stem cell-based approaches have received considerable attention as a potential means of treatment, although it remains to be determined whether stem cells can ameliorate memory dysfunction, a devastating component of these disorders. We generated a transgenic mouse model in which the tetracycline-off system is used to regulate expression of diphtheria toxin A chain. After induction, we find progressive neuronal loss primarily within the hippocampus, leading to specific impairments in memory. We find that neural stem cells transplanted into the brain after neuronal ablation survive, migrate, differentiate and, most significantly, improve memory. These results show that stem cells may have therapeutic value in diseases and conditions that result in memory loss.
\end{abstract}

Key words: transgenic; stem cells; neuronal death; functional recovery; memory; ablation

\section{Introduction}

Neuronal loss is a common feature of many neurological maladies that affect the brain including traumatic brain injury (TBI), stroke, and Alzheimer disease (Morrison and Hof, 1997; Bramlett and Dietrich, 2004). Transplantation of neural stem cells offers a promising therapeutic strategy for minimizing the functional damage that manifests in these disorders (Cao et al., 2002; Lindvall et al., 2004; Oliveira and Hodges, 2005; Lindvall and Kokaia, 2006; Vora et al., 2006). Transplanted cells may serve as a reservoir, providing trophic support to surviving cells and synapses, or they may actually replace and partially repopulate damaged areas. However, the ultimate measure of the therapeutic utility of stem cells in the brain is recovery of function, particularly in regard to the ability to rescue memory impairments. Although several previous studies report locomotor recovery resulting from stem cell transplant after brain or spinal cord injury (Cummings et al., 2005; Bernreuther et al., 2006; Yasuhara et al., 2006; Ziv et al., 2006), it remains to be established whether stem cell therapy is a viable approach for the treatment of neurological conditions resulting in memory impairment.

Research on brain injury and recovery, particularly as it relates to cognition, has been hampered in part by the complexity and variability inherent in many of the commonly used animal mod-

Received April 11, 2007; revised Sept. 12, 2007; accepted Sept. 14, 2007

This work was supported by the National Institute on Aging (F.M.L.). T.R.Y. was supported by National Institutes of Health Medical Scientist Training Program Grant GM08620, and M.B.-J. was supported by a California Institute for Regenerative Medicine postdoctoral scholar award. We thank Drs. Henry Klassen (Children's Hospital of Orange County, Orange, (A) and Michael Young (Harvard Medical School, Boston, MA) for the GFP NSC cell line. We are

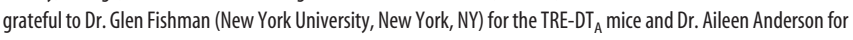
advice and use of her laboratory's stereology setup.

Correspondence should be addressed to Frank M. LaFerla, Department of Neurobiology and Behavior, University of California, Irvine, 1109 Gillespie Neuroscience Building, Irvine, CA 92697-4545. E-mail: laferla@uci.edu. DOI:10.1523/JNEUROSCI.1627-07.2007

Copyright $\odot 2007$ Society for Neuroscience ～0270-6474/07/2711925-09\$15.00/0 els. Most methods of inducing brain injury are invasive, requiring surgical procedures that disrupt the blood-brain barrier by mechanical means, percussion or aspiration of brain matter (Jarrard, 2002). Pharmacologically induced lesions may produce secondary effects on other systems and also typically require impaling the brain to achieve regional specificity, and ischemic models deprive entire brain regions of nutrients and oxygen, often resulting in variable damage to multiple regions, and are also complicated by reperfusion effects or a mixed population of cell death, especially at the ischemic penumbra (Jarrard, 2002; Carmichael, 2005). Moreover, these approaches for injuring the brain typically cannot target precise cell populations, and widespread regional damage limits the evaluation of complex cognitive tasks.

Here, we report the development of a unique transgenic model of neuronal injury in which the tetracycline (tet)inducible promoter system is used to temporally and spatially regulate the expression of diphtheria toxin A-chain $\left(\mathrm{DT}_{\mathrm{A}}\right.$ ), a potent cytotoxin for eukaryotic cells (Kochi and Collier, 1993). Regional specificity is conferred by utilization of the calciumcalmodulin kinase II $\alpha$ (CaMKII $\alpha)$-regulatory region, thereby enabling selective and targeted ablation of forebrain neurons. We show that CaMKII $\alpha$-expressing neurons in the forebrain are selectively vulnerable in a graded manner (i.e., CA1 region $>$ cortex). Notably, the precision of this system renders it possible to restrict the amount of neuronal loss and evaluate cognitive function both before and after a neuronally targeted and inducible brain lesion. This system is ideal for exploring the therapeutic potential of neural stem cells. Toward this end, we transplanted stem cells in the brains of $C a M / T e t-D T_{A}$ mice with neuronal loss and found long-term survival of stem cells, differential migration patterns, and differentiation into mature cell types. Neural stemcell transplantation also increases hippocampal synaptic density and decreases neuronal death. Most significantly, we find that 
transplantation of neural stem cells produces a significant restoration of memory. Our findings provide enticing evidence that stem cell-based therapies may offer a viable approach for the treatment of common neurological disorders and memory dysfunction.

\section{Materials and Methods \\ Animals}

Double transgenic mice were generated by crossing CaMKII $\alpha$ - $t$ TA mice (B6/CBA) to tetracycline-responsive element (TRE)-DT $T_{A}$ mice (B6/ CBA). Because we were unable to breed CaMKII $\alpha$ - $t$ TA mice to homozygosity, both lines were maintained separately and crossed together to generate $\mathrm{CaM} / \mathrm{Tet}-\mathrm{DT} \mathrm{T}_{A}$ mice hemizygous for each transgene. To prevent induction of $\mathrm{DT}_{\mathrm{A}}$, mating pairs and offspring were maintained on 2000 parts per gram doxycycline chow (Research Diets, New Brunswick, NJ) and switched at weaning to $2 \mathrm{mg} / \mathrm{ml}$ doxycycline (Sigma, St. Louis, MO) supplemented with $5 \%$ sucrose in deionized, filtered drinking water. Mice were genotyped by PCR, using primer pairs $5^{\prime}$-CGCATTAGAGCTGCTTAATG-3' and 5' -TCGCGATGACTTAGTAAAGC-3' for the CaMKII $\alpha$-tTA transgene and $5^{\prime}$-TCTTCGTACCACGGGACTAA- ${ }^{\prime}$ and $5^{\prime}$-CCGCAGCGTCGTATTTATTG-3' for the TRE-DT A $_{\mathrm{A}}$ transgene. Induction of $\mathrm{DT}_{\mathrm{A}}$ expression in adult bigenic mice was conducted by administering water without supplemental doxycycline. All procedures were performed in accordance with the regulations of the Institutional Animal Care and Use Committee of the University of California, Irvine.

\section{Reverse-transcriptase PCR}

RNA was isolated from hemibrains that were homogenized in GuCNS solution ( $4 \mathrm{~m}$ guanidine thiocyanate, $0.5 \%$ Sarkosyl, $0.025 \mathrm{M} \mathrm{Na}$ citrate, $100 \mathrm{~mm} \beta$-mercaptoethanol) and phenol/chloroform was extracted. RNA samples were treated with RQ1 RNase-free DNase (Promega, Madison, WI) for $15 \mathrm{~min}$ in the presence of RNasin (Promega), followed by reverse transcription with Superscript II RT using Oligo dT primers (Invitrogen, Carlsbad, $\mathrm{CA}$ ). $\mathrm{DT}_{\mathrm{A}}$ was amplified using the above listed primers and PCR.

\section{Tissue processing}

Mice used for histological analysis were either perfused or killed by carbon dioxide inhalation. For perfusions, mice were anesthetized with nembutal sodium solution (Ovation Pharmaceuticals, Deerfield, IL) and perfused transcardially at a rate of $12 \mathrm{ml} / \mathrm{min}$ first with PBS followed by $4 \%$ paraformaldehyde in $0.01 \mathrm{~m}$ phosphate buffer, $\mathrm{pH}$ 7.4. Brains were postfixed in $4 \%$ paraformaldehyde for $48 \mathrm{~h}$, and sectioned at $40 \mu \mathrm{m}$ by vibratome (Pelco, Redding, CA). Sections were subsequently stored in PBS with $0.02 \%$ sodium azide.

\section{Immunohistochemistry and differentiation analysis}

For terminal deoxynucleotidyl transferase-mediated biotinylated UTP nick end labeling (TUNEL), sections were incubated with $0.02 \mathrm{mg} / \mathrm{ml}$ proteinase $\mathrm{K}$ (Promega) at $37^{\circ} \mathrm{C}$ for $15 \mathrm{~min}$. Sections were incubated with terminal transferase reaction mix (Roche, Indianapolis, IN) at $37^{\circ} \mathrm{C}$ for $45 \mathrm{~min}$ and developed with diaminobenzidine substrate using the avidinbiotin horseradish peroxidase system (Vector Laboratories, Burlingame, CA). For anti-NeuN (1:10,000; Millipore, Temecula, CA) staining, tissues were first quenched with $3 \% \mathrm{MeOH} /$ hydrogen peroxide for $30 \mathrm{~min}$, then preincubated with $0.1 \%$ Triton-X and $3 \%$ normal serum and developed on the second day with SG-1 (Vector Laboratories).

For immunofluorescence, sections were first blocked with 3\% normal serum with $2 \%$ BSA and $0.1 \%$ Triton-X in TBS, then incubated with primary antibody in blocking solution overnight at $4^{\circ} \mathrm{C}$. They were then rinsed and incubated with secondary Alexa Fluor-conjugated antibodies (1:200; Invitrogen) in block, rinsed, and mounted in Fluoromount G (Southern Biotech, Birmingham, AL). Primary antibodies were as follows: anti-GFAP (1:500; DAKO, Glostrup, Denmark), anti-NeuN (1:10,000; Millipore), anti-NG2 chondroitin sulfate proteoglycan (1:200; Millipore), and anti-green fluorescent protein (GFP; 1:2000; Millipore). For anti-CNPase (1:20,000; Sigma), signal amplification was performed with the Tyramide Signal Amplification System
(PerkinElmer, Waltham, MA). To determine percentage of differentiation in stem cell injected animals, tissues double-stained for either NeuN/GFP (neurons), GFAP/GFP (astrocytes), or NG2/GFP (oligodendrocyte precursors) were analyzed at the 4.5 month posttransplant time point. Because of the difficulty of consistently labeling cell bodies with a mature oligodendrocyte stain, counts were taken with NG2, a marker of oligodendrocyte precursors (Polito and Reynolds, 2005). Counts of GFP + cells at the dentate gyrus (including the molecular layers) were first taken using a $10 \times 10$ counting grid at $10 \times$ magnification, and then colocalization of GFP with each marker $($ NeuN, GFAP, or NG2) was assessed at $20 \times$ magnification. The percentage differentiation was thus number of cells colocalized with the given marker divided by total number of GFP + cells. Counts were taken bilaterally on $n=5$ animals and then averaged. For colocalization of labels in Figure 5, cells were double labeled with immunofluorescent markers and analyzed by confocal microscopy (Olympus, Tokyo, Japan) using the LaserSharp 2000 program. $Z$-scans were conducted in $0.5 \mu \mathrm{m}$ steps with Kallman $n=2$. Lambda strobing and was used in all scans to reduce nonspecific signal.

\section{ScionImage quantification of NeuN staining}

For each group of mice ( $n=5$ per group), similar sections were stained for NeuN in the same staining set. Three consecutive nonoverlapping pictures were taken across the CA1 subfield at $40 \times$ magnification. All pictures were taken in one sitting with no change in illumination. Pictures were imported into ScionImage software program (National Institutes of Health, Bethesda, MD) and the threshold was set at 100 for all pictures. Extraneous cells outside of the CA1 subfield were removed and pixel intensity was determined.

\section{Stereological cell counts}

All unbiased stereological assessments were performed using StereoInvestigator software (MicroBrightField, Williston, VT). For each stereological evaluation, an optical fractionator probe was used to estimate mean cell numbers and the Cavalieri principle was used to determine volume of each brain region. Guard zones were set at $10 \%$ of measured thickness with a minimum $12 \mu \mathrm{m}$ optical dissector height. Contour tracing to delineate brain regions was done using a $4 \times$ objective and counting was performed using a $100 \times$ oil objective. The counting was done in every 12th section (40 $\mu \mathrm{m}$ coronal sections) using $n=5$ animals per group. A $25 \times 25 \mu \mathrm{m}$ counting frame was used in all stereological evaluations and all counts were performed in only one brain hemisphere. Quantification of cell loss in CA1 and dentate gyrus of $30 \mathrm{~d}$ induced, $20 \mathrm{~d}$ induced, and noninduced animals was performed on five Nissl-stained sections extending anteriorly $-1.28 \mathrm{~mm}$ from bregma and using a $150 \times$ $150 \mu \mathrm{m}$ sampling grid. Data are shown as mean estimated cell numbers for this stereological assessment. Stereological evaluation of NeuN and GFP in mice injected with stem cells was done on series of sections throughout the region where GFP-labeled cells could be seen between bregma $-1.28 \mathrm{~mm}$ and bregma $-2.92 \mathrm{~mm}$. For GFP-labeled cell counting, a $500 \times 500 \mu \mathrm{m}$ sampling grid was used to assess the entire hippocampus and the entire cortex. Counting of NeuN-positive cells was done in CA1 and dentate gyrus using $200 \times 200 \mu \mathrm{m}$ sampling grid.

\section{Optical density quantification}

Two sections per animal $(n=7-9)$ were immunofluorescently labeled in parallel for the presynaptic vesicle protein synapsin-1 (1:500; Calbiochem, San Diego, CA) following standard protocols. Slides were coded and images captured by a blinded observer using a Bio-Rad (Hercules, CA) Radiance 2100 confocal microscope and identical scan settings. Grayscale images were inverted and optical density was quantified using ImageJ software. Briefly, regions of interest (ROIs) including the stratum radiatum of CA1 and the polymorph layer of the dentate gyrus were defined in each image and average pixel intensity was measured from each ROI. Measurements of white matter provided background levels which were subtracted from ROI measurements. Pixel intensity from two sections per animal were averaged and then animal pixel intensity was compared. 


\section{Endogenous neurogenesis levels}

5-Bromo-2'-deoxyuridine (Sigma) was reconstituted in water with PBS at $\mathrm{pH} 7.4$ at a concentration of $5 \mathrm{mg} / \mathrm{ml}$. Mice were weighed at the start of the experiment and volumes of injectate were calculated for $50 \mathrm{mg} / \mathrm{kg}$ drug/animal weight. The labeling paradigm used was described by Jin et al. (2004). Briefly, bromodeoxyuridine (BrdU) was injected twice a day for the last four $\mathrm{d}$ of a $20 \mathrm{~d}$ induction period, with each injection separated by a minimum of $8 \mathrm{~h}$. BrdU was administered twice on the last day, and then mice were returned to a dox water regimen to abrogate $\mathrm{DT}_{\mathrm{A}}$ expression. Mice were perfused and killed 1 month after the last injection. To assess neurogenesis levels, the percentage of NeuN+BrdU+ colocalizing cells in the granule cell layer of the dentate gyrus was determined by confocal analysis. Each cell at the granule cell layer in a series of $4-6$ sections spaced $240 \mu \mathrm{m}$ apart $\left(\mathrm{CaM} / \mathrm{Tet}-\mathrm{DT} \mathrm{T}_{A}\right.$ mice) was imaged on a confocal microscope at $60 \times$ or higher magnification. For determining colocalization, BrdU + cells were scanned for colocalization of NeuN. If NeuN did not colocalize definitively throughout the entire length of the $\mathrm{BrdU}+$ nucleus, then the cell was not considered colocalized. If the staining was ambiguous as to colocalization or no colocalization, the cell was excluded. Settings for $Z$-scans were maintained throughout the entire series as follows: green, iris, 1.5; gain, 39.0; offset, -70.5 ; laser, 6.0 ; red, iris, 1.7; gain, 21.2; offset, -5.4 ; laser, 17.0). All scans were performed with $\lambda$ strobbing and Kallman $n=2$ stop to reduce nonspecific background and at a speed of 166 lines per second. $Z$-stacks were taken at 0.5 $\mu \mathrm{m}$ steps. The percentage of colocalization was determined by dividing the number of BrdU $+\mathrm{NeuN}+$ cells by the total number of (nonambiguous) colocalized and noncolocalized BrdU+ cells.

\section{NSC maintenance and isolation}

Mouse enhanced GFP-expressing neural stem cells were grown in DMEM/F12 with Glutamax and epidermal growth factor-2 (EGF-2) (20 $\mathrm{ng} / \mathrm{ml}$ ). On the day of the surgery, cells at postnatal day 14 (P14)-P17 were harvested, triturated, filtered and resuspended in $1 \times$ HBSS with EGF-2 $(20 \mathrm{ng} / \mathrm{ml})$ at a concentration of 50,000 cells/ $\mu$ l. Aliquots of vehicle and cell suspension were stored in sterile-O-ring-sealed tubes on ice until they were removed for injection/transplantation, at which point they were triturated and drawn up for injection in Flexifil tapertip syringes (World Precision Instruments, Sarasota, FL).

\section{Surgeries and NSC transplantation}

Adult $C a M / T e t-D T_{A}$ mice $4-6$ months of age were induced by withdrawal from doxycycline water. After $25 \mathrm{~d}$ on a regular food and water diet, they were returned to doxycycline-supplemented water to abrogate expression of diphtheria toxin. Eleven days after the start of abrogation, stem cell surgeries were performed. Mice were anesthetized with Avertin $(0.6 \mathrm{ml} / 25 \mathrm{~g}$ body weight $)$. After appropriate tests for depth of anesthesia, each mouse was stereotactically injected bilaterally with $2 \mathrm{ul}$ of either vehicle or neural stem cells (NSCs) at a rate of $1 \mu \mathrm{l} / \mathrm{min}$. The syringe was allowed to remain in place for $2 \mathrm{~min}$ after the injection to allow diffusion of the injectate into the surrounding tissue. Separate flexifil syringes were used for NSC injections and vehicle injections, and syringes were thoroughly rinsed with deionized water, and then buffer after each injection. Mice were randomized to receive either vehicle or NSCs injections. Coordinates as determined by the mouse brain atlas (Franklin and Paxinos, 1997) were in relation to bregma as follows: anteroposterior, $-2.06 \mathrm{~mm}$; mediolateral (M/L), $\pm 1.75 \mathrm{~mm}$; dorsoventral (D/V), $-1.75 \mathrm{~mm}$. For induced mice, because of hippocampal shrinkage with induction, coordinates were altered slightly based on pilot studies so the injection site would be comparable with the noninduced hippocampal site with coordinates $\mathrm{M} / \mathrm{L} \pm 2.00 \mathrm{~mm}$ and $\mathrm{D} / \mathrm{V}-1.85 \mathrm{~mm}$. Surgery sites were sealed with bone wax, sutured, closed with tissue-mend, and topical antibiotic ointment was applied to the site. Mice were allowed to recover consciousness on heating pads, and then housed singly for a few weeks until the surgery site had healed. At this point, females were group housed and males were paired with nonbreeding females to prevent negative effects of long-term isolation on future behavior.

\section{Behavioral procedures}

Habituation. Each behavioral group consisted of $n=8-13$ animals. Briefly, two Plexiglas rectangular chambers $18 \times 9.5 \times 10$ inches were used for the behavioral tests. Mice were handled for 3 consecutive days (days 1-3), habituated for $15 \mathrm{~min}$ in groups of three or less (day 4), then for $10 \mathrm{~min}$ with one mouse per cage (day 5 ), and $5 \mathrm{~min}$ with one mouse per cage (day 6). We followed well documented behavioral procedures for object and place memory tests (Mumby et al., 2002). All mice received only one behavioral test per day. On day 7, half of the mice were given place tests, and half were given object tests. On day 9, these were switched so that the half receiving the place test on day 7 received object tests on day 9 and vice versa for the other half of the groups. In all tests, two mice were run simultaneously in adjacent cages separated by an opaque barrier. Novel or displaced objects were counterbalanced in terms of location in the cage. Objects were plastic or metal and $\sim 1.5$ inches in height. Different sets of objects were used for each behavioral task at the 1 month and 3 month post-transplantation time points to eliminate the possibility of retained memory for objects. However, all pairs of objects (novel and familiar) were tested on nonexperimental mice to rule out innate preference for objects in any given pairing. The chamber was cleaned with $70 \%$ ethanol (EtOH) between cages of mice and all objects were washed thoroughly with $70 \% \mathrm{EtOH}$ before each trial. All exploratory segments and tests were videotaped for scoring purposes. If an animal did not explore both objects during the training phase, it was not scored during the test phase. In the scoring procedure, because some mice exhibited freezing or fearful behavior on introduction to the chamber, scoring did not start until the mice physically moved from their initial starting position: always in the corner closest to the familiar object. Exploration counted if the mouse's head was within one inch of the object with neck extended and vibrissae moving. Simple proximity, chewing, or standing on the object did not count as exploration.

Object recognition. Each mouse was placed in the chamber with two identical objects spaced $\sim 12$ inches apart. The animals were allowed to explore the objects for $5 \mathrm{~min}$. After a $5 \mathrm{~min}$ retention interval in which the animal was returned to its home cage, the mouse was place back in the chamber with the previously exposed object and a novel object for a 3 min probe test.

Place recognition. Each mouse was placed in the chamber with two identical objects spaced by $\sim 12$ inches apart. After a 5 min exploration, then a 5 min retention interval in their home cage, mice were returned to the chamber in which one of the two objects was displaced from its original position.

\section{Statistical analysis}

Data were analyzed by $t$ test or ANOVA for multiple comparisons unless otherwise indicated and considered significant at $p \leq 0.05$.

\section{Results \\ Inducible transgenic model with temporally and spatially progressive neuronal loss}

To genetically lesion selective populations of neurons in the brain, we generated a transgenic model with regulatable expression of $\mathrm{DT}_{\mathrm{A}}$, a potent cytotoxin for eukaryotic cells (Kochi and Collier, 1993). The tet-inducible promoter system enables temporal and spatial regulation, where regional specificity is conferred by utilization of the CaMKII $\alpha$-regulatory region. Withdrawal of doxycycline from the drinking water of the mice allows transactivator binding to the TRE of a second transgene, which controls expression of $\mathrm{DT}_{\mathrm{A}}$ (Fig. $1 A$ ). Double transgenic mice $\left(C a M /\right.$ Tet- $\left.D T_{A}\right)$ were established by crossing TRE-DT $T_{A}$ mice (Lee et al., 1998) with CaMKII $\alpha$ - $t$ TA mice (Mayford et al., 1996).

Tight control of $\mathrm{DT}_{\mathrm{A}}$ expression is crucial for limiting the extent of neuronal loss and for avoiding leakiness during noninduced periods. We evaluated $\mathrm{DT}_{\mathrm{A}}$ transgene expression using reverse transcriptase (RT)-PCR, a highly sensitive readout, and 
find that $\mathrm{DT}_{\mathrm{A}}$ expression is first apparent at $8 \mathrm{~d}$ postinduction, but not in noninduced mice (Fig. 1B) (data not shown). Abrogating $\mathrm{DT}_{\mathrm{A}}$ expression, which is important for limiting the magnitude of the lesion, can be achieved within $7 \mathrm{~d}$ of reintroducing dox (Fig. 1C). Collectively, the data indicate that transgene expression is very tightly regulated, and that $\mathrm{DT}_{\mathrm{A}}$ expression can be induced and subsequently abrogated with precision in the brains of adult mice.

We next histopathologically evaluated the consequences of progressive $\mathrm{DT}_{\mathrm{A}}$ expression in the brain. Noninduced double-transgenic mice showed no evidence of neuronal damage by hematoxylin and eosin (H\&E) staining or by NeuN, a marker for mature neuronal nuclei (Fig. $2 A-D$ ). Depending on the length of induction, we found that pyramidal neurons in the CA1 subfield are the first neuronal cell type to die in response to $\mathrm{DT}_{\mathrm{A}}$ induction, with substantial degeneration from this region apparent by $20 \mathrm{~d}$ postinduction (Fig. $2 \mathrm{E}-\mathrm{H}$ ). Thirty days of induction leads to the marked destruction of virtually all neurons in the CA1 subfield (Fig. $2 I-L$ ). Hence, longer periods of $\mathrm{DT}_{\mathrm{A}}$ induction lead to progressively greater damage. Dying neurons appeared pyknotic, and were readily apparent by their highly eosinophilic cytoplasm and condensed nuclei that were dark and basophilic after H\&E staining (Fig. $2 F, J$ ).

We further quantified neuronal number by evaluating brain sections stained with NeuN, using optical densitometry. As expected, longer periods of transgene induction $(20,25$, and $30 \mathrm{~d}$ ) led to significant reductions in NeuN optical density at the CA1 subfield when compared with noninduced controls (Fig. 2M). To quantitatively assess these changes, we performed unbiased stereology at the CA1 subfield and dentate gyrus in noninduced and $30 \mathrm{~d}$ induced mice. Consistent with NeuN optical density measurements, stereology showed a significant reduction in neuronal number at the CA1 subfield, as well as at the dentate gyrus of the hippocampus (Fig. $2 \mathrm{~N}$ ). TUNEL and Fluorojade B staining (supplemental Fig. 1, available at www.jneurosci.org as supplemental material) further confirmed the same pattern of cell death as that seen with $\mathrm{H} \& \mathrm{E}$ and NeuN. Thus, $\mathrm{DT}_{\mathrm{A}}$ expression in an inducible transgenic context provides an effective means for ablating select populations of neurons in the CNS.

In addition to the progressive neuronal loss within the hippocampus, there is also a regional progression within the brain. We find that loss from the hippocampus initiates before loss from cortical areas, as assessed by Fluorojade B. At $15 \mathrm{~d}$ of induction, there is defined loss from CA1 regions of the hippocampus, but no apparent corresponding loss from cortical structures (Fig. $3 A, B)$. At $20 \mathrm{~d}$ of induction, cell death is substantial in the CA1 region, but sparse at the cortical area (Fig. $3 C, D$ ). By $25 \mathrm{~d}$ of induction, there is severe loss from the CA1 region, as well as consistent loss from cortical subfields (Fig. $3 E, F$ ). Hence, longer periods of $\mathrm{DT}_{\mathrm{A}}$ induction lead to progressively greater damage, and further, analysis indicates there is a spatial pattern of cell ablation such that loss from the CA1 region initiates before cortical loss occurs.

\section{Endogenous neurogenesis is not affected by a targeted lesion of hippocampal neurons}

Neuronal loss has also been shown to affect neurogenesis in certain models, such as ischemia, traumatic brain injury, and epilepsy (Gould and Tanapat, 1997; Parent et al., 1997; Jin et al., 2001). To determine the impact of selective ablation of a subpopulation of neurons from the hippocampus on levels of endogenous neurogenesis, we induced $C a M / T e t-D T_{A}$ mice for $20 \mathrm{~d}$, at which time CA1 loss is apparent, and labeled newborn cells with the thymidine analog, BrdU. To assess levels of neurogenesis, $\mathrm{DT}_{\mathrm{A}}$ expression was abrogated by the readdition of doxycycline to the water regimen, and the percentage of the newly generated cells expressing NeuN was determined at 1 month after BrdU labeling. There was no significant change in levels of endogenous neurogenesis at the subgranular zone and granule cell layers of the dentate gyrus after cell loss from the CA1 region in CaM/ Tet-DT $T_{A}$ mice (supplemental Fig. 2, available at www.jneurosci. org as supplemental material).

\section{Memory impairments correlate with progressive neuronal loss}

To define the effect of selective neuronal loss on cognitive function, we tested $C a M /$ Tet-DT $T_{A}$ mice for object recognition and place memory following well described methods (Mumby et al., 2002). Place memory is considered to be mostly hippocampaldependent in that it requires memory for the original spatial location (Parkinson et al., 1988; Eichenbaum, 2000). In contrast, object recognition relies mostly on cortical areas, including the perirhinal cortex (Buffalo et al., 1998; Brown and Aggleton, 2001; Winters et al., 2004). We tested CaM/Tet-DT $T_{A}$ mice at two different induction time points on these tasks. At $20 \mathrm{~d}$ of induction, cell loss from the hippocampus is most significant from the CA1 region with minimal loss from cortical areas (Fig. 3C,D). These mice have a significant deficit for place memory, the hippocampal-dependent spatial task, and are not significantly impaired relative to noninduced controls on object recognition memory (Fig. 4). After $30 \mathrm{~d}$ of induction, a time point at which lesioning in the cortex as well as hippocampus is significant, we found that $C a M /$ Tet- $D T_{A}$ mice were significantly impaired in both object and place memory compared with noninduced con- 


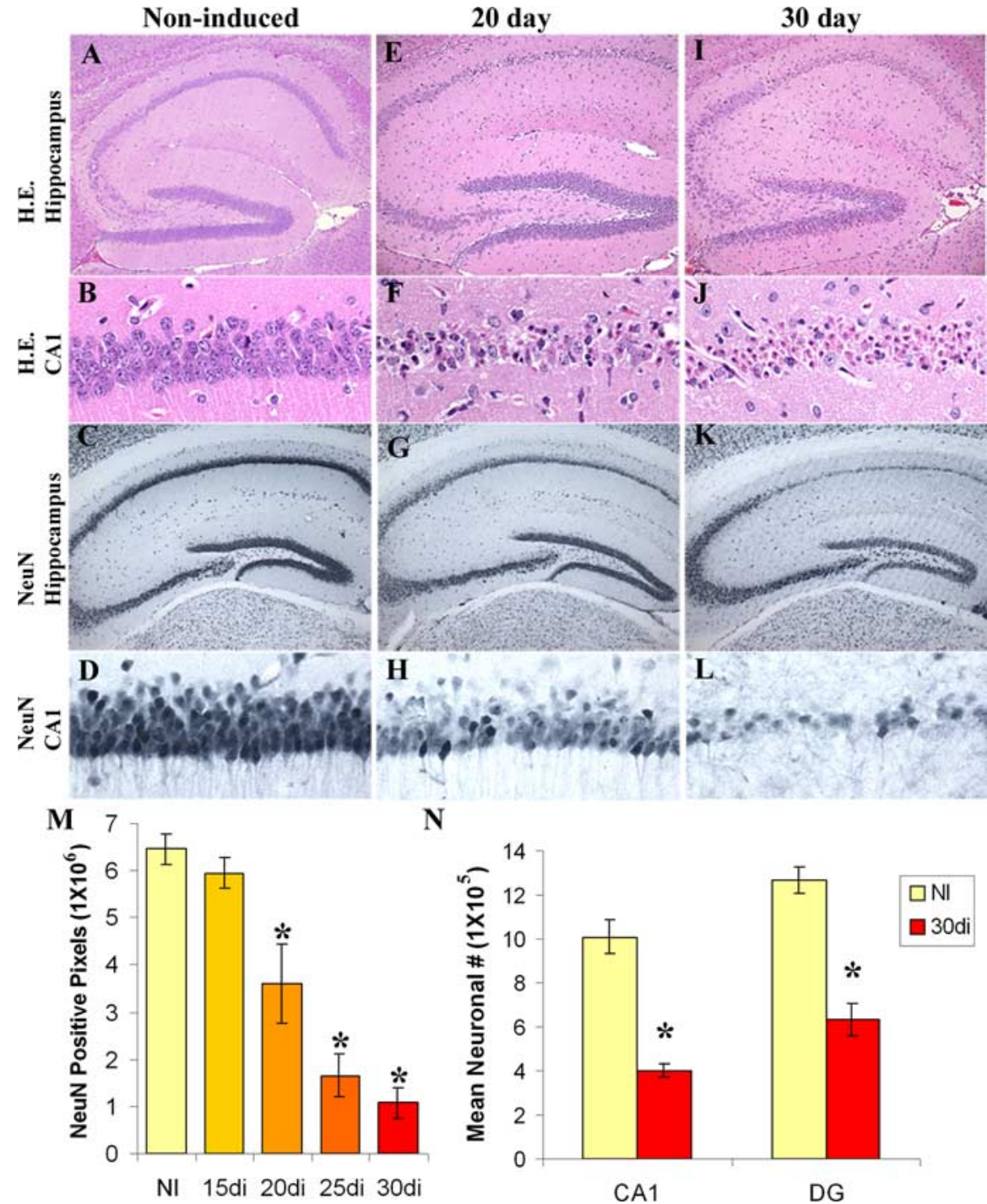

Figure 2. Induction of $\mathrm{DT}_{\mathrm{A}}$ expression results in neuronal loss from hippocampal regions. $\boldsymbol{A}-\boldsymbol{D}$, No neuronal loss is apparent in noninduced mice as demonstrated by $H \& E$ and NeuN staining. $\boldsymbol{E}-\boldsymbol{H}$, Twenty days of induction leads to significant loss from the CA1 subfield. $I-L$, Thirty days of induction leads to widespread neuronal loss from the hippocampus. $M$, Loss of NeuN staining from the CA1 subfield is significantly decreased ${ }^{*}$ ) from noninduced controls (NI) by $20 \mathrm{~d}$ of induction (20di) and exhibits further decline with 25 (25di) and $30 \mathrm{~d}$ of induction (30di) (ANOVA, $F_{(4,21)}=32.77, p<0.0001$, post hoc Bonferroni). $\boldsymbol{N}$, Significant loss of neurons $(*)$ from both the $C A 1$ region and dentate gyrus at $30 \mathrm{~d}$ of induction is confirmed by unbiased stereological methods [CA1, $t$ test, $p=0.0052$; dentate gyrus (DG), $t$ test, $p=0.0009$.

trols (Fig. 4). Notably, induced mice were still able to perform the object recognition task above chance levels, indicating that they retain some memory for the object, but they were completely impaired on the place memory test. As expected, there was no significant difference in baseline performance of noninduced $C a M / T e t-D T_{A}$ mice and nontransgenic mice on either the object or place recognition tasks, and both groups performed well above chance levels (Fig. 4). Thus, there is no detectable effect of the $\mathrm{CaM} / \mathrm{Tet}-\mathrm{DT}_{A}$ transgene on performance of these tasks in noninduced animals.

\section{Transplanted NSCs survive, migrate, and differentiate into mature types within the lesioned brain}

To determine whether NSC transplantation is a viable approach for improving the memory deficits, we transplanted mouse NSCs expressing enhanced GFP or vehicle into the hippocampi of $C a M / T e t-D T_{A}$ mice after $25 \mathrm{~d}$ of induction at which time point $\sim 74 \%$ of their CA1 neurons were ablated (Fig. 2M). As a control, we also implanted NSCs into noninduced mice. Behavioral testing was conducted at two time points post-transplant and brains were then analyzed histologically (Fig. 5A).

The mouse NSCs were derived from enhanced GFP-expressing mice at postnatal day 1 and express GFP under control of the chicken $\beta$-actin promoter (Okabe et al., 1997). Properties of these NSCs have been well documented previously (Mizumoto et al., 2003) and we further confirmed that these they express nestin, as well as markers of immature neurons including TuJ1 (neuronal class III $\beta$-tubulin) and DCX (doublecortin) in vitro (data not shown).

In previous studies, local environmental factors have been shown to affect cell migration and differentiation (Hoehn et al., 2002; Kelly et al., 2004). To determine the response of transplanted NSCs to the microenvironment created by selective neuronal ablation, we conducted a histopathological characterization of these mice at 4.5 months post-transplantation. We find that NSCs survive well out to this time point. Interestingly, striking differences in the distribution and migration of NSCs were readily apparent between the brains of induced and noninduced mice. In noninduced mice, NSCs migrate toward cortical and white matter areas, including the corpus callosum and travel as far as the optic nerve (data not shown), whereas in mice with induced neuronal loss, NSCs remain within the hippocampus but fail to migrate away from the lesion into the cortex and white matter (Fig. $5 B)$. To quantify these distributions, we used stereology to determine the number of GFP + cells in the hippocampus and cortex of induced and noninduced mice. Overall, a significantly greater number of NSCs are present in the cortex of noninduced mice (Fig. 5C,D), whereas no significant difference was evident within the hippocampus (Fig. 5D). Thus, the microenvironment created by the $\mathrm{DT}_{\mathrm{A}}$-induced lesion may inhibit survival of GFP NSCs, but also significantly decreases migration from the site of the lesion. Importantly, NSCs are capable of differentiating into mature cell types, including neurons (Fig. $5 E$ ), astrocytes (Fig. $5 F$ ), and mature oligodendrocytes (Fig. 5G). Confocal analysis indicated percentages of differentiation as follows: $1.78 \pm$ $0.515 \%$ neurons, $15.39 \pm 6.76 \%$ astrocytes, and $17.07 \pm 3.49 \%$ oligodendrocyte precursors.

NSCs improve hippocampal-dependent memory and increase synaptic density and neuronal number

The ultimate measure of the utility of stem cell transplantation into the brain is functionality. To determine the effects of NSC implantation on memory, mice were tested behaviorally at one and 3 months after transplantation. These time points were se- 


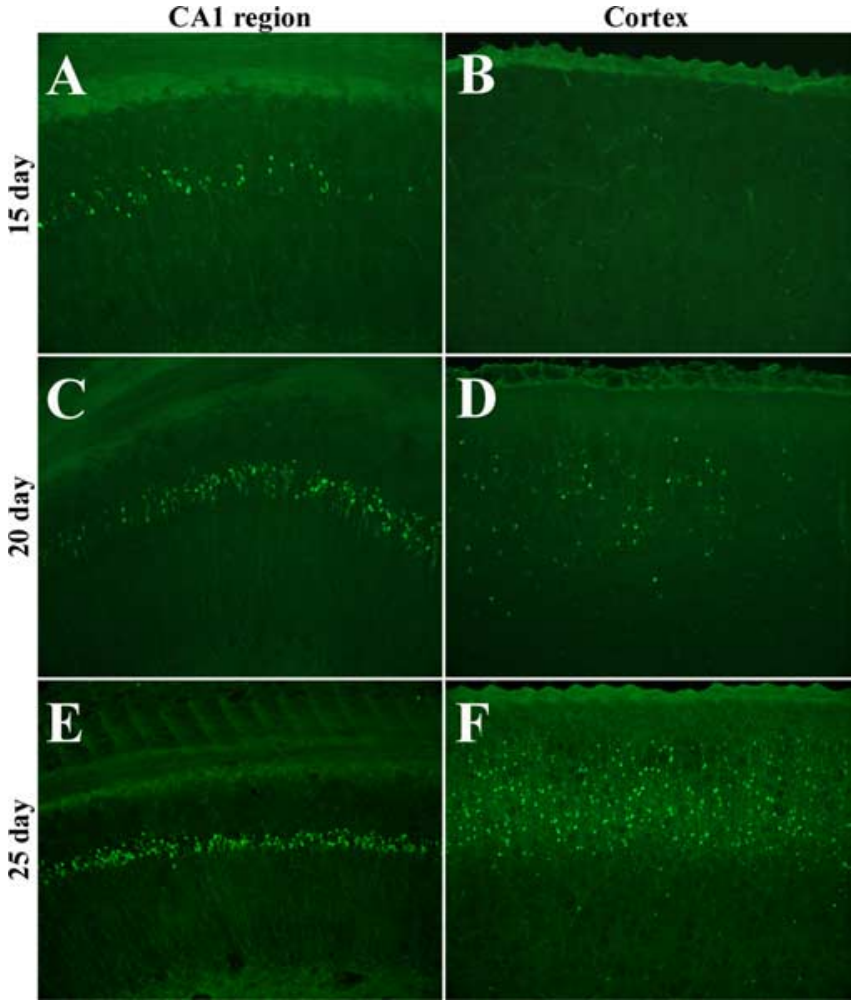

Figure 3. Length of induction affects the regional progression of neuronal loss. $A, B$, Fifteen days of induction results in loss localized to the $C A 1$ region of the hippocampus $(A)$, without corresponding loss from cortical regions $(\boldsymbol{B})$. $\boldsymbol{C}-\boldsymbol{F}$, Twenty days of induction results in substantial loss from the $C A 1$ subfield $(\boldsymbol{C})$ with sparse loss from cortical regions $(\boldsymbol{D})$, whereas $30 \mathrm{~d}$ of induction results in widespread loss from the CA1 region $(\boldsymbol{E})$ and cortex $(\boldsymbol{F})$.

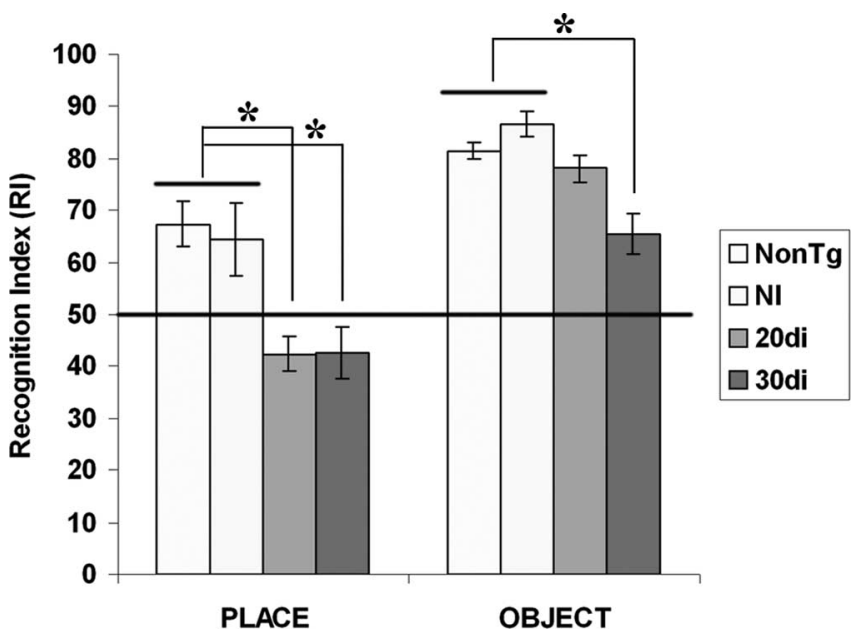

Figure 4. Hippocampal-dependent memory impairments. In mice induced for $20 \mathrm{~d}$ (20di) in which the majority of loss occurs within the CA1 region of the hippocampus, hippocampaldependent place memory is significantly impaired $\left({ }^{*}\right)$, but cortical-dependent object memory is not significantly different from noninduced controls (NI). However, in mice induced for $30 \mathrm{~d}$ (30di), both place and object memory are significantly impaired $(*)$, which is consistent with the extent of neuronal loss in both hippocampal and cortical areas. Noninduced CaM/Tet-DT $T_{A}$ mice are not significantly different from nontransgenic control mice. (place: one-way ANOVA, $p=$ $0.035 ; \mathrm{NI}$ vs 20di, $p=0.0187 ; \mathrm{NI}$ vs $30 \mathrm{di}, p<0.01 ; \mathrm{NI}$ vs NonTg, $p=0.9452 ;$ object: one-way ANOVA, $p<0.0008$; NI vs 20di, $p>0.05$; NI vs 30di, $p<0.01$; NI vs NonTg, $p>0.05$ ).

lected to allow sufficient time for differentiation and integration. At 1 month post-transplant, although the results were not significantly different, we found that NSC-transplanted mice outperform their vehicle-injected counterparts in both object and place memory ( $p=0.1583, p=0.1311$, respectively) (Fig. $6 A$ ). However, by 3 months post-transplantation, mice that received neural stem cells exhibit significantly better memory for recognition of place, a mostly hippocampal-dependent task, than vehicle-injected controls ( $p=0.0429$ ) (Fig. 6B). Object recognition, which is more dependent on cortical regions, is not significantly improved in NSC-transplanted mice $(p=0.2137)$. Thus, in mice with a hippocampal lesion, transplanting NSCs into the damaged area significantly improves memory for a hippocampaldependent task, whereas no effect is apparent for object recognition, a primarily cortical-dependent task.

Stem cells have been postulated to exert their effects in different ways, one of which is through production of neurotrophins (Llado et al., 2004; Yasuhara et al., 2006). Indeed, the GFP NSCs used in this study produce brain-derived neurotrophic factor (BDNF) in vitro (data not shown). Thus, we speculated that NSCs may enhance synaptic plasticity or neuronal survival via a trophic mechanism. To determine whether stem cell transplant affects synaptic plasticity in $C a M / T e t-D T_{A}$ mice, we compared optical densities of the presynaptic vesicle protein synapsin in lesioninduced mice that had received either NSC or vehicle 4.5 months before being killed. Two areas critically involved in hippocampal circuitry were quantified including the stratum radiatum of CA1 and the polymorph layer of the dentate gyrus. Notably, a significant increase in synaptic density was detected within the stratum radiatum of CA1 in mice that received NSC-transplants versus vehicle-injected controls (Fig. 6C,D). Synaptic density at the dentate gyrus, although not significantly different, demonstrated a similar trend (Fig. 6C,D). In addition to modulating synaptic density, neurotrophins can also prevent the loss of vulnerable neuronal populations after varying insults (for review, see Tuszynski and Gage, 1995). Indeed, stereological assessment revealed that significantly more neurons survive in both the CA1 region and the dentate gyrus after a lesion when NSCs are transplanted (Fig. 6E,F).

\section{Discussion}

Here, we demonstrate the feasibility of using bigenic mice to inducibly destroy a select population of neurons in the adult mammalian CNS. By using the CaMKII $\alpha$ promoter to drive expression of the tet transactivator, inducible $\mathrm{DT}_{\mathrm{A}}$ expression was limited to forebrain neurons, and significantly, we showed that the system is titratable such that CA1 pyramidal neurons are ablated first without causing overt damage to other brain regions. In addition to precise spatial regulation, we further demonstrate tight temporal regulation, enabling one to bypass expression during critical developmental periods. Likewise, our study indicates that there was no $\mathrm{DT}_{\mathrm{A}}$ leakiness, as no cell loss was observed in noninduced bigenic mice (supplemental Fig. 1, available at www. jneurosci.org as supplemental material). Furthermore, there is regional progression to the lesion, such that the CA1 field of the hippocampus is affected before cortical regions and the dentate gyrus. Last, we demonstrate tight control not only over transgene induction, but we further prove that expression can be readily abrogated, thereby controlling the extent of the lesion. We conclude that inducible control of $\mathrm{DT}_{\mathrm{A}}$ offers many advantages for ablating distinct populations within the CNS, and by altering the transcriptional promoter used to drive expression of the tet transactivator, this system can be readily adapted for knocking out other neuronal subtypes.

The focal nature of the neuronal loss in our transgenic model is demonstrated quite clearly on a histopathological level. We find that the loss of CA1 neurons leads to specific memory deficits 

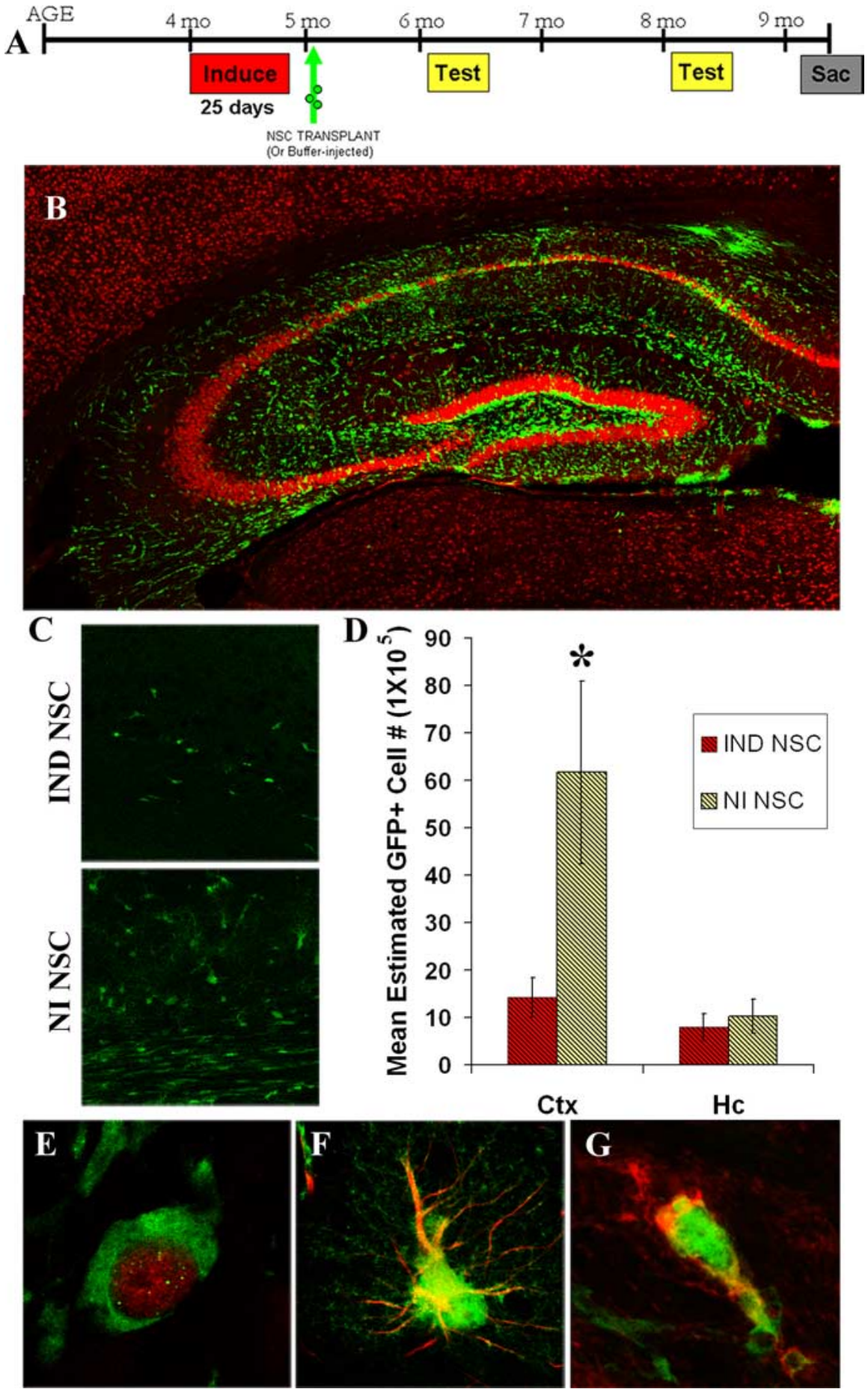

Figure 5. NSC transplants survive for at least 4.5 months and migrate and differentiate into neurons and glia. $A$, Paradigm of NSC transplantation in induced CaM/Tet-DT $T_{A}$ mice. $B$, Hippocampi at 4.5 months post-transplant of induced mouse showing NSCS (green) and neurons stained with NeuN (red). C, GFP NSCs transplanted into the hippocampus show migration differences based on microenvironment, with significantly more GFP + cells migrating into cortical and white matter areas in noninduced mice compared with induced mice. D, Stereological quantification of GFP NSCs show significantly more GFP NSCs in the cortex (Ctx) of noninduced NSC-injected mice ${ }^{*}$, cortex, $t$ test, $\left.p=0.05\right)$. $\boldsymbol{E}, \boldsymbol{F}$, GFP NSCs colocalize with NeuN (D), a marker of mature neuronal nuclei, GFAP-immunoreactive astrocytes $(\boldsymbol{E})$, and CNPase $(\boldsymbol{F})$, a marker for mature oligodendrocytes.

on a hippocampal-dependent task, but notably did not cause impairment on a cortically dependent task. By taking advantage of the ability to progressively lesion regions of the brain with increasing induction times, we are also able to induce to a time point at which both hippocampal and cortically dependent behavioral tasks are impaired. Thus, cytotoxin expression targeted to a specific neuronal subpopulation results in region-specific behavioral deficits and underlines the utility of this model.

In this study, we used our model to determine whether transplantation of neural stem cells would lead to improved performance on a short-term memory task. This question is significant, as many neurological disorders are marked by profound cognitive impairments, and it is believed that once neuronal loss occurs, the impairments would not be reversible. Notably, most animal models of neurodegenerative disorders show minimal, if any, cell loss. In contrast, the novel model we present here allows us to specifically address whether memory can be improved after extensive and selective neuronal loss.

Regarding the neural stem cell transplantation, there are several significant findings relevant to survival, migration, and differentiation. First, we find that transplanted NSCs survive for at least 4.5 months (the longest time point we examined). Second, we find that stem cells tend to be distributed more widely in noninduced mice, where they migrated from their initial site of transplantation in the hippocampus through white matter areas including the corpus callosum and fimbria fornix and into the deep layers of the cerebral cortex. It is tempting to speculate that the migratory differences in mice with and without neuronal loss may be attributable to the presence of some attractant or repellent cue to which neural stem cells respond. Also, it is interesting to note that there are, overall, a greater number of GFP + cells present in the brains of noninduced mice, than in induced mice. It is possible that the microenvironment created by selective neuronal loss resulted in greater cell death of NSCs. Finally, we confirmed the multipotency of these GFP+ neural stem cells and showed that they differentiate into neurons, astrocytes, and oligodendrocytes in vivo. Although we find a small percentage of GFP + neurons, it is also possible that GFP expression is downregulated after differentiation. One group has found that retrovirus- and lentivirus-transfected neural progenitor cells transplanted into the spinal cord lose GFP expression after differentiation (Vroemen et al., 2005). If this is the case, then the number of differentiated cells may have been underestimated.

A common endpoint for studies examining functional recovery after stem cell transplants has been restoration of motor function (Cummings et al., 2005; Bernreuther et al., 2006; Yasuhara et al., 2006; Ziv et al., 2006). In contrast, few studies have examined a cognitive endpoint, and of these, the results are conflicting. A few have found improvement (Toda et al., 2001; Shear et al., 
A 1 month post-transplant
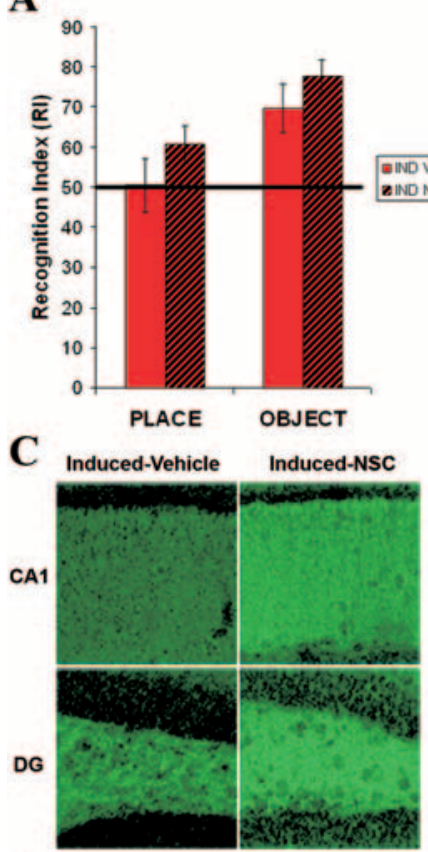

$\mathbf{E}$

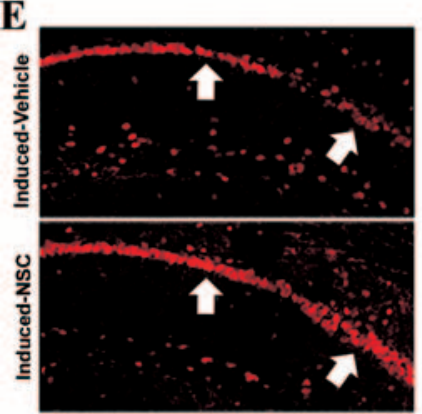

B

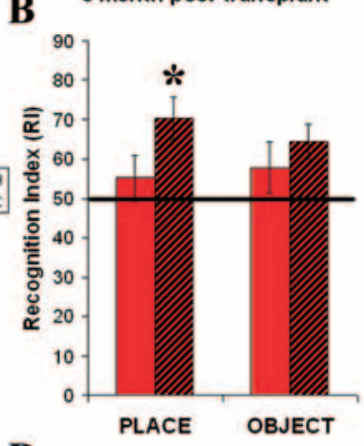

D

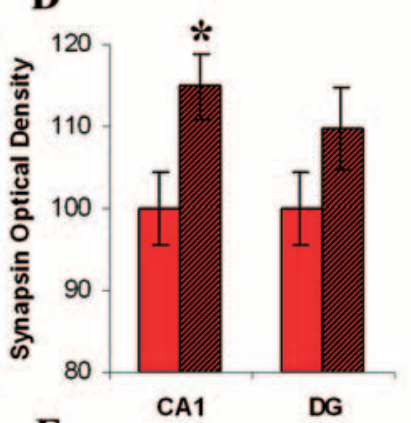

F

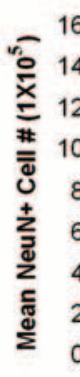

CA1

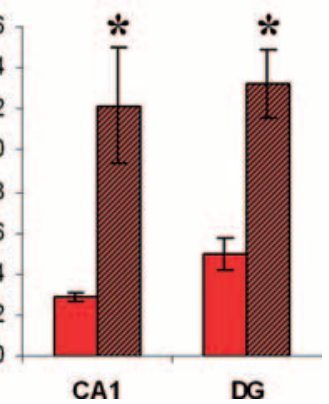

Figure 6. NSCs improve hippocampal-dependent memory and increase synaptic density and neuronal number. Place and object memory in CaM/Tet-DT $T_{A}$ mice induced for $25 \mathrm{~d}$ at one month $(\boldsymbol{A} ; p=0.1583 ; p=0.1311)$ and three months $(\boldsymbol{B})$ post-transplant. At 3 months, induced mice with NSC transplants (IND NSC) show significant improvement in the hippocampal-dependent spatial task $\left({ }^{*} p=0.0429\right)$ compared with induced vehicle injected controls (IND VEH) ( $p=0.2137)$. C, Synaptic density in induced-vehicle injected vs inducedNSC injected mice at the stratum radiatum of the CA1 region and the polymorph layer of the dentate gyrus. $D$, A significant increase in synaptic density $\left(^{*}\right)$ is seen at the stratum radiatum of the CA1 subfield of NSC-injected induced mice [CA1 $t$ test, $p=0.0316$; dentate gyrus (DG), $t$ test, $p=0.151]$. $\boldsymbol{E}$, There is also an increase in NeuN + cells at the CA1 layer of induced NSC-injected mice compared with their vehicle-injected counterparts. $\boldsymbol{F}$, This difference is significant $\left(^{*}\right)$ at both the CA1 region and the dentate gyrus (CA1 $t$ test, $p=0.0035 ; \mathrm{DG}, t$ test, $p=$ 0.0099).

2004; Gao et al., 2006); however, other groups have found no improvement (Veizovic et al., 2001; Hoane et al., 2004) or have confusingly found improvement without concomitant survival of cells (Jeltsch et al., 2003). Here, we show that neural stem cells transplanted into the hippocampus of a transgenic model with targeted neuronal loss are able to improve short-term memory on a spatial task in a time-dependent manner. Furthermore, the fact that the stem cells localize to the hippocampus in induced mice is consistent with the selective improvement seen on the hippocampal-dependent task, but not on the cortically dependent task. Our study is unique in two distinct ways. First, previous studies examined transplants in models with lesions induced through traumatic processes or ischemic processes, both of which are variable in the region(s) affected and negatively impact all cell types (neuronal, glial, endothelial, etc.). This is in direct contrast to the focal and cell-specific nature of neuronal loss in our CaM/ Tet-DT $T_{A}$ mice. Second, our behavioral paradigm was chosen for its dual advantage of examining cognitive function on two tasks primarily dependent on differing brain regions (Mumby et al., 2002), hippocampus for place memory (Parkinson et al., 1988; Eichenbaum, 2000) and perirhinal cortex for object recognition (Buffalo et al., 1998; Brown and Aggleton, 2001; Winters et al., 2004), and also for the fact that it makes use of the natural tendency of the mouse to explore objects perceived as novel and is thus less stressful than a water maze paradigm (Ennaceur and Delacour, 1988; Mumby et al., 2002).

In our study, we find that it takes 3 months for a memory effect to manifest. It has been shown that transplanted stem cells take over a month in vivo to develop the electrophysiological responsiveness of mature neurons (Auerbach et al., 2000; Englund et al., 2002), form long projecting axons in the brain, and develop excitatory and inhibitory synapses (Englund et al., 2002). Thus, it is interesting that we find only a trend toward improvement at 1 month and significant improvement of memory at 3 months.

Trophic mechanisms are likely to contribute to the improvement of memory in our model. Increases in the neurotrophic factor BDNF occur after brain injury (Kokaia et al., 1998) and BDNF has also been shown to upregulate synapsin (Causing et al., 1997). The levels of synapsin we see in induced NSC transplanted mice significantly outstrip those seen in lesioned vehicleinjected controls at the CA1 region of the hippocampus, and are consistent with neurotrophin-induced sprouting. Further, it is likely that neurotrophins contribute to the neuronal sparing effect we see at both the dentate gyrus and CA1 region of NSC transplanted mice resulting in memory improvement. Last, although we do not see an increase in endogenous neurogenesis with lesioning, another possible therapeutic avenue, a neurotrophin-mediated augmentation of endogenous neurogenesis, is plausible and has, in fact, been described by other groups (Zigova et al., 1998; Yoshimura et al., 2001).

Our study is the first to show that stem cells can help improve short-term memory after a focal brain lesion targeted specifically to a neuronal subpopulation. Our approach is differentiated from other studies in which the lesion induces damage not only to neurons but also to other surrounding cells including glia and endothelial cells. Because our genetic approach leads to specific loss of a select neuronal population, our findings indicate that the transplanted stem cells compensate for the lost neuronal function, and not by modulating other cell types in the damaged area. These findings provide strong and compelling evidence that neural stem cell transplantation may offer a viable therapeutic approach to treat patients suffering from diseases and conditions that involve neuronal loss and memory impairment.

\section{References}

Auerbach JM, Eiden MV, McKay RD (2000) Transplanted CNS stem cells form functional synapses in vivo. Eur J Neurosci 12:1696-1704.

Bernreuther C, Dihne M, Johann V, Schiefer J, Cui Y, Hargus G, Schmid JS, Xu J, Kosinski CM, Schachner M (2006) Neural cell adhesion molecule L1-transfected embryonic stem cells promote functional recovery after excitotoxic lesion of the mouse striatum. J Neurosci 26:11532-11539.

Bramlett HM, Dietrich WD (2004) Pathophysiology of cerebral ischemia and brain trauma: similarities and differences. J Cereb Blood Flow Metab 24:133-150.

Brown MW, Aggleton JP (2001) Recognition memory: what are the roles of the perirhinal cortex and hippocampus? Nat Rev Neurosci 2:51-61.

Buffalo EA, Reber PJ, Squire LR (1998) The human perirhinal cortex and recognition memory. Hippocampus 8:330-339. 
Cao Q, Benton RL, Whittemore SR (2002) Stem cell repair of central nervous system injury. J Neurosci Res 68:501-510.

Carmichael ST (2005) Rodent models of focal stroke: size, mechanism, and purpose. NeuroRx 2:396-409.

Causing CG, Gloster A, Aloyz R, Bamji SX, Chang E, Fawcett J, Kuchel G, Miller FD (1997) Synaptic innervation density is regulated by neuronderived BDNF. Neuron 18:257-267.

Cummings BJ, Uchida N, Tamaki SJ, Salazar DL, Hooshmand M, Summers R, Gage FH, Anderson AJ (2005) Human neural stem cells differentiate and promote locomotor recovery in spinal cord-injured mice. Proc Natl Acad Sci USA 102:14069-14074.

Eichenbaum H (2000) A cortical-hippocampal system for declarative memory. Nat Rev Neurosci 1:41-50.

Englund U, Bjorklund A, Wictorin K, Lindvall O, Kokaia M (2002) Grafted neural stem cells develop into functional pyramidal neurons and integrate into host cortical circuitry. Proc Natl Acad Sci USA 99:17089-17094.

Ennaceur A, Delacour J (1988) A new one-trial test for neurobiological studies of memory in rats. 1: Behavioral data. Behav Brain Res 31:47-59.

Franklin KBJ, Paxinos G (1997) The mouse brain in stereotaxic coordinates. San Diego: Academic.

Gao J, Prough DS, McAdoo DJ, Grady JJ, Parsley MO, Ma L, Tarensenko YI, Wu P (2006) Transplantation of primed human fetal neural stem cells improves cognitive function in rats after traumatic brain injury. Exp Neurol 201:281-292.

Gould E, Tanapat P (1997) Lesion-induced proliferation of neuronal progenitors in the dentate gyrus of the adult rat. Neuroscience 80:427-436.

Hoane MR, Becerra GD, Shank JE, Tatko L, Pak ES, Smith M, Murashov AK (2004) Transplantation of neuronal and glial precursors dramatically improves sensorimotor function but not cognitive function in the traumatically injured brain. J Neurotrauma 21:163-174.

Hoehn M, Kustermann E, Blunk J, Wiedermann D, Trapp T, Wecker S, Focking M, Arnold H, Hescheler J, Fleischmann BK, Schwindt W, Buhrle C (2002) Monitoring of implanted stem cell migration in vivo: a highly resolved in vivo magnetic resonance imaging investigation of experimental stroke in rat. Proc Natl Acad Sci USA 99:16267-16272.

Jarrard LE (2002) Use of excitotoxins to lesion the hippocampus: update. Hippocampus 12:405-414.

Jeltsch H, Yee J, Aloy E, Marques Pereira P, Schimchowitsch S, Grandbarbe L, Caillard S, Mohier E, Cassel JC (2003) Transplantation of neurospheres after granule cell lesions in rats: cognitive improvements despite no longterm immunodetection of grafted cells. Behav Brain Res 143:177-191.

Jin K, Minami M, Lan JQ, Mao XO, Batteur S, Simon RP, Greenberg DA (2001) Neurogenesis in dentate subgranular zone and rostral subventricular zone after focal cerebral ischemia in the rat. Proc Natl Acad Sci USA 98:4710-4715.

Jin K, Galvan V, Xie L, Mao XO, Gorostiza OF, Bredesen DE, Greenberg DA (2004) Enhanced neurogenesis in Alzheimer's disease transgenic (PDGF-APPSw,Ind) mice. Proc Natl Acad Sci USA 101:13363-13367.

Kelly S, Bliss TM, Shah AK, Sun GH, Ma M, Foo WC, Masel J, Yenari MA, Weissman IL, Uchida N, Palmer T, Steinberg GK (2004) Transplanted human fetal neural stem cells survive, migrate, and differentiate in ischemic rat cerebral cortex. Proc Natl Acad Sci USA 101:11839-11844.

Kochi SK, Collier RJ (1993) DNA fragmentation and cytolysis in U937 cells treated with diphtheria toxin or other inhibitors of protein synthesis. Exp Cell Res 208:296-302.

Kokaia Z, Andsberg G, Yan Q, Lindvall O (1998) Rapid alterations of BDNF protein levels in the rat brain after focal ischemia: evidence for increased synthesis and anterograde axonal transport. Exp Neurol 154:289-301.

Lee P, Morley G, Huang Q, Fischer A, Seiler S, Horner JW, Factor S, Vaidya D, Jalife J, Fishman GI (1998) Conditional lineage ablation to model human diseases. Proc Natl Acad Sci USA 95:11371-11376.

Lindvall O, Kokaia Z (2006) Stem cells for the treatment of neurological disorders. Nature 441:1094-1096.

Lindvall O, Kokaia Z, Martinez-Serrano A (2004) Stem cell therapy for human neurodegenerative disorders-how to make it work. Nat Med [Suppl] 10:S42-S50.
Llado J, Haenggeli C, Maragakis NJ, Snyder EY, Rothstein JD (2004) Neural stem cells protect against glutamate-induced excitotoxicity and promote survival of injured motor neurons through the secretion of neurotrophic factors. Mol Cell Neurosci 27:322-331.

Mayford M, Bach ME, Huang YY, Wang L, Hawkins RD, Kandel ER (1996) Control of memory formation through regulated expression of a CaMKII transgene. Science 274:1678-1683.

Mizumoto H, Mizumoto K, Shatos MA, Klassen H, Young MJ (2003) Retinal transplantation of neural progenitor cells derived from the brain of GFP transgenic mice. Vision Res 43:1699-1708.

Morrison JH, Hof PR (1997) Life and death of neurons in the aging brain. Science 278:412-419.

Mumby DG, Gaskin S, Glenn MJ, Schramek TE, Lehmann H (2002) Hippocampal damage and exploratory preferences in rats: memory for objects, places, and contexts. Learn Mem 9:49-57.

Okabe M, Ikawa M, Kominami K, Nakanishi T, Nishimune Y (1997) "Green mice" as a source of ubiquitous green cells. FEBS Lett 407:313-319.

Oliveira Jr AA, Hodges HM (2005) Alzheimer's disease and neural transplantation as prospective cell therapy. Curr Alzheimer Res 2:79-95.

Parent JM, Yu TW, Leibowitz RT, Geschwind DH, Sloviter RS, Lowenstein DH (1997) Dentate granule cell neurogenesis is increased by seizures and contributes to aberrant network reorganization in the adult rat hippocampus. J Neurosci 17:3727-3738.

Parkinson JK, Murray EA, Mishkin M (1988) A selective mnemonic role for the hippocampus in monkeys: memory for the location of objects. J Neurosci 8:4159-4167.

Polito A, Reynolds R (2005) NG2-expressing cells as oligodendrocyte progenitors in the normal and demyelinated adult central nervous system. J Anat 207:707-716.

Shear DA, Tate MC, Archer DR, Hoffman SW, Hulce VD, Laplaca MC, Stein DG (2004) Neural progenitor cell transplants promote long-term functional recovery after traumatic brain injury. Brain Res 1026:11-22.

Toda H, Takahashi J, Iwakami N, Kimura T, Hoki S, Mozumi-Kitamura K, Ono S, Hashimoto N (2001) Grafting neural stem cells improved the impaired spatial recognition in ischemic rats. Neurosci Lett 316:9-12.

Tuszynski MH, Gage FH (1995) Maintaining the neuronal phenotype after injury in the adult CNS. Neurotrophic factors, axonal growth substrates, and gene therapy. Mol Neurobiol 10:151-167.

Veizovic T, Beech JS, Stroemer RP, Watson WP, Hodges H (2001) Resolution of stroke deficits following contralateral grafts of conditionally immortal neuroepithelial stem cells. Stroke 32:1012-1019.

Vora N, Jovin T, Kondziolka D (2006) Cell transplantation for ischemic stroke. Neurodegener Dis 3:101-105.

Vroemen M, Weidner N, Blesch A (2005) Loss of gene expression in lentivirus- and retrovirus-transduced neural progenitor cells is correlated to migration and differentiation in the adult spinal cord. Exp Neurol 195:127-139.

Winters BD, Forwood SE, Cowell RA, Saksida LM, Bussey TJ (2004) Double dissociation between the effects of peri-postrhinal cortex and hippocampal lesions on tests of object recognition and spatial memory: heterogeneity of function within the temporal lobe. J Neurosci 24:5901-5908.

Yasuhara T, Matsukawa N, Hara K, Yu G, Xu L, Maki M, Kim SU, Borlongan CV (2006) Transplantation of human neural stem cells exerts neuroprotection in a rat model of Parkinson's disease. J Neurosci 26:12497-12511.

Yoshimura S, Takagi Y, Harada J, Teramoto T, Thomas SS, Waeber C, Bakowska JC, Breakefield XO, Moskowitz MA (2001) FGF-2 regulation of neurogenesis in adult hippocampus after brain injury. Proc Natl Acad Sci USA 98:5874-5879.

Zigova T, Pencea V, Wiegand SJ, Luskin MB (1998) Intraventricular administration of BDNF increases the number of newly generated neurons in the adult olfactory bulb. Mol Cell Neurosci 11:234-245.

Ziv Y, Avidan H, Pluchino S, Martino G, Schwartz M (2006) Synergy between immune cells and adult neural stem/progenitor cells promotes functional recovery from spinal cord injury. Proc Natl Acad Sci USA 103:13174-13179. 